\title{
The parton cascade BAMPS with the improved Gunion-Bertsch matrix element
}

\author{
Florian Senzel \\ Institut für Theoretische Physik, Johann Wolfgang Goethe-Universität, Max-von-Laue-Str. 1, \\ 60438 Frankfurt am Main, Germany \\ E-mail: senzeleth.physik.uni-frankfurt.de
}

\section{Oliver Fochler}

Institut für Theoretische Physik, Johann Wolfgang Goethe-Universität, Max-von-Laue-Str. 1, 60438 Frankfurt am Main, Germany

E-mail: fochlereth.physik.uni-frankfurt.de

\section{Jan Uphoff}

Institut für Theoretische Physik, Johann Wolfgang Goethe-Universität, Max-von-Laue-Str. 1, 60438 Frankfurt am Main, Germany

E-mail: uphoffeth.physik.uni-frankfurt.de

\section{Christian Wesp}

Institut für Theoretische Physik, Johann Wolfgang Goethe-Universität, Max-von-Laue-Str. 1, 60438 Frankfurt am Main, Germany

E-mail: cwespeth.physik.uni-frankfurt.de

\section{Zhe Xu}

Department of Physics, Tsinghua University, Beijing 100084, China

E-mail: xuzhe@mail.tsinghua.edu.cn

\section{Carsten Greiner ${ }^{* \dagger}$}

Institut für Theoretische Physik, Johann Wolfgang Goethe-Universität, Max-von-Laue-Str. 1, 60438 Frankfurt am Main, GermanyUniversity of Frankfurt

E-mail: carsten.greinereth.physik.uni-frankfurt.de

\begin{abstract}
Aiming at a simultaneous description of both the hard and the soft regime of ultra-relativistic heavy-ion collisions at RHIC and LHC, we present our recent progress in studying the nuclear modification factor $R_{A A}$, the momentum imbalance of reconstructed di-jets $A_{J}$, and the elliptic flow $v_{2}$ within the partonic transport model BAMPS (Boltzmann Approach to Multi-Parton Scatterings). By numerically solving the relativistic Boltzmann equation while using both elastic and inelastic perturbative QCD interactions together with an improved Gunion Bertsch matrix element and a running coupling, BAMPS allows the full 3+1D simulation of the quark-gluon plasma at the microscopic level. We show that when fixing the parameter $X_{L P M}$ resulting from the effective modeling of the Landau-Pomeranchuk-Migdal effect, we find not only a good description of $R_{A A}$ and $A_{J}$, but also a sizable elliptic flow is built up within the partonic phase.
\end{abstract}

52 International Winter Meeting on Nuclear Physics

27 - 31 January 2014

Bormio, Italy 


\section{Introduction}

When heavy nuclei collide at ultra-relativistic energies, they create a system of hot and dense matter. Due to the enormous available energy densities within these collisions, quasi-free quarks and gluons represent the relevant degrees of freedom. Therefore the produced medium is commonly called the "quark-gluon plasma". Experiments at both the Relativistic Heavy-Ion Collider (RHIC) at BNL and the Large Hadron Collider (LHC) at CERN show that the created medium exhibits interesting properties [1,2]: While high energy particles traversing the medium are quenched, the system shows at the same time a collective behavior similar to a nearly perfect liquid.

Among the most prominent observables for quantifying these properties are the nuclear modification factor, $R_{A A}$, and the elliptic flow, $v_{2}$. While $R_{A A}$, defined as

$$
R_{A A}=\frac{\mathrm{d}^{2} N_{\mathrm{AA}} / \mathrm{d} p_{T} \mathrm{~d} y}{N_{\mathrm{bin}} \mathrm{d}^{2} N_{\mathrm{pp}} / \mathrm{d} p_{T} \mathrm{~d} y},
$$

measures the suppression of inclusive particle yields compared to scaled $p+p$ collisions, the elliptic flow $v_{2}$, defined in terms of the second Fourier coefficient of the azimuthal particle distribution, gives insight to the collectivity of the medium.

Furthermore, due to the enormous collision energy of the LHC and thereby increased production cross section of high $p_{t}$ partons, the separation between the momenta of the traversing partons and the underlying background medium become more distinct with the result that energy loss studies in terms of reconstructed jets become feasible. By studying reconstructed jets within heavy-ion collisions, both the ATLAS [3] as well as the CMS experiment $[4,5]$ reported the measurement of an enhanced number of events with an asymmetric pair of reconstructed back-to-back jets in comparison with $\mathrm{p}+\mathrm{p}$ events. One observable for describing this asymmetry in reconstructed jet momenta is the momentum imbalance

$$
A_{J}\left(p_{t ; 1}, p_{t ; 2}\right)=\frac{p_{t ; 1}-p_{t ; 2}}{p_{t ; 1}+p_{t ; 2}},
$$

where $p_{t ; 1}\left(p_{t ; 2}\right)$ is the transverse momentum of the leading (subleading) jet- the reconstructed jet with the highest (second highest) transverse momentum per event. Already in $p+p$ collisions, where no medium creation is expected, the QCD vacuum splitting processes of the initial partons with high virtuality lead to a non-zero $A_{J}$ distribution. The further enhancement of unbalanced events in heavy-ion collisions is the result of different in-medium energy loss of the two partons within the created bulk medium, which is a consequence of a non-central spatial production point of the initial di-jet pair.

Although jet quenching and bulk phenomena are both commonly attributed to the partonic phase of the heavy-ion collision, a simultaneous understanding on the microscopic level remains a challenge $[6,7,8,9,10,11,12]$. In this paper we report on our progress in understanding the QGP within the partonic transport model Boltzmann Approach to Multi-Parton Scatterings (BAMPS). Based on cross sections calculated in $\mathrm{pQCD}$, soft and hard particles are treated on the same footing in a common framework. While we take explicitly the running of the coupling into account, we

\footnotetext{
* Speaker.

${ }^{\dagger}$ A footnote may follow.
} 


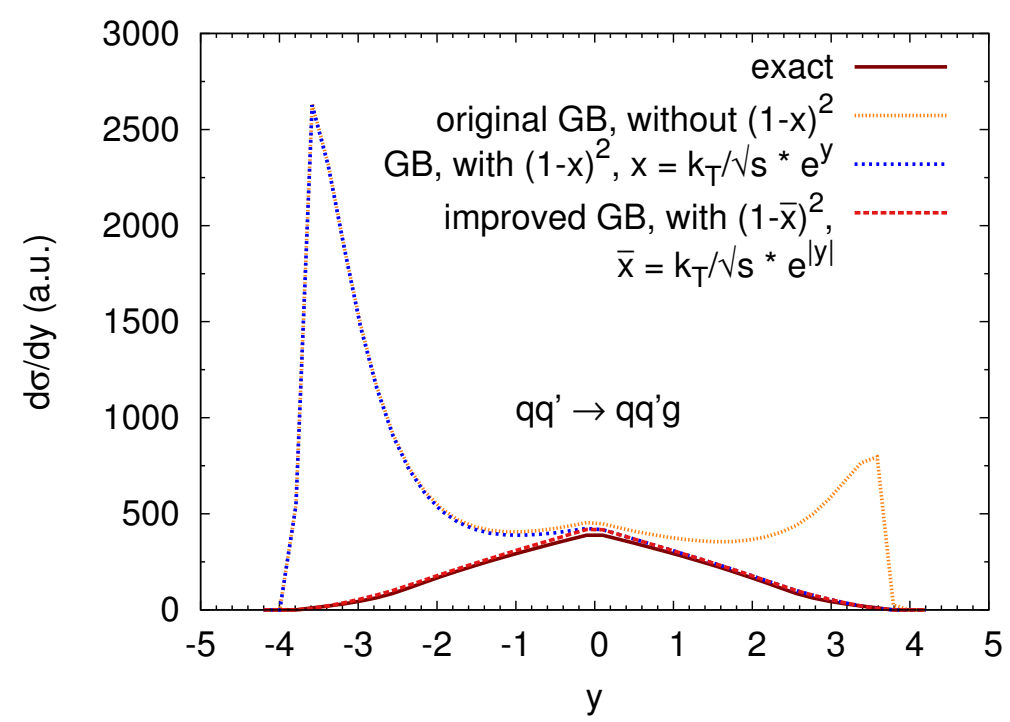

Figure 1: Differential cross section $\mathrm{d} \sigma / \mathrm{d} y$ for the process $q q^{\prime} \rightarrow q q^{\prime} g$ calculated with the exact [14], the GB [15], the GB with $(1-x)^{2}$ and $x=\frac{k_{\perp}}{\sqrt{s}} \mathrm{e}^{y}$, and the improved GB with $(1-\bar{x})^{2}$ matrix element [16].

study not only the energy loss of highly energetic particles, but also the collective behavior of the bulk medium.

\section{The partonic transport model BAMPS}

The partonic transport model Boltzmann Approach to Multi-Parton Scatterings (BAMPS) [10, 13] describes the full 3+1D evolution of both the QGP medium as well as high energy particles traversing it by numerically solving the relativistic Boltzmann equation,

$$
p^{\mu} \partial_{\mu} f(\vec{x}, t)=\mathscr{C}_{22}+\mathscr{C}_{2 \leftrightarrow 3},
$$

for on-shell partons, quarks and gluons, and perturbative quantum-chromodynamics (pQCD) interactions. To this end, a stochastical modeling of the collision probabilities together with a testparticle ansatz is employed.

Within BAMPS, both elastic $2 \rightarrow 2$ scattering processes calculated in leading-order pQCD, like e.g. $g g \rightarrow g g$, and inelastic $2 \leftrightarrow 3$ interactions, like e.g. $g g \leftrightarrow g g g$, based on the GunionBertsch (GB) approximation [15] are included. Recent studies [16] show that the "original GunionBertsch" matrix element [15] is not fully valid in the forward and backward rapidity region of the emitted gluon. To this end, an improved GB matrix element,

$$
\left|\overline{\mathscr{M}}_{X \rightarrow Y+g}\right|^{2}=\left|\overline{\mathscr{M}}_{X \rightarrow Y}\right|^{2} P_{g}
$$

with

$$
P_{g}=48 \pi \alpha_{S}\left(k_{\perp}^{2}\right)(1-\bar{x})^{2}\left[\frac{\mathbf{k}_{\perp}}{k_{\perp}^{2}}+\frac{\mathbf{q}_{\perp}-\mathbf{k}_{\perp}}{\left(\mathbf{q}_{\perp}-\mathbf{k}_{\perp}\right)^{2}+m_{D}^{2}\left(\alpha_{S}\left(k_{\perp}^{2}\right)\right)}\right]^{2}
$$


was proposed that shows a good agreement with the exact pQCD matrix element over the considered phase space region, cf. Fig. 2 [16]. Within the GB approximation the matrix element factorizes into an elastic part $\left|\mathscr{M}_{X \rightarrow Y}\right|$ and a probability $P_{g}$ for emitting a gluon, where $k_{\perp}$ and $q_{\perp}$ are the transverse momentum of the emitted and internal gluons, respectively. The longitudinal momentum fraction $\bar{x}$ carried away by the emitted gluon can be related to the gluon rapidity in the center-of-mass system of the respective microscopic collision by $\bar{x}=\frac{k_{\perp}}{\sqrt{s}} e^{|y|}$.

Analogously to the implementation of heavy quarks in BAMPS $[17,18]$, we also recently implemented a running coupling $\alpha_{s}(t)$ for the light parton sector. By setting the scale of the coupling to the momentum transfer of the considered channel, the running coupling is evaluated for each collision at the microscopic level.

For modeling the Landau-Pomeranchuk-Migdal (LPM) effect, which is an important quantum effect within a partonic QCD medium, an effective cutoff function $\theta\left(\lambda-X_{L P M} \tau_{f}\right)$ in the radiative matrix elements is used, where $\lambda$ is the mean free path of the radiating particle and $\tau_{f}$ the gluon formation time. For $X_{L P M}=1$, this effectively allows only independent inelastic scatterings, while $X_{L P M}=0$ leads to no LPM suppression at all. A more sophisticated implementation of the LPM effect, where also some interference processes occur, should lead to a parameter in the intermediate region $0<X_{L P M}<1$. For now, we treat $X_{L P M}$ as a parameter and fix its value based on the $R_{A A}$ of neutral pions at RHIC, cf. Sec. 3. Any further divergences resulting from the integration of the pQCD matrix elements are cured by a screening Debye mass, which is dynamically computed on the basis of the current quark and gluon distribution [10].

\section{Results}

As established in [19, 20, 21, 22, 23, 24] the QGP simulated within previous BAMPS studies employing the "original Gunion-Bertsch" matrix element together with a fixed coupling showed a collectivity that resulted in an elliptic flow that was in agreement with data. However, the same interactions driving the elliptic flow also led to a suppression of jets that was too strong in comparison with the $R_{A A}$ of neutral pions at RHIC. In the following we present our recent results for both observables and, additionally, the momentum imbalance $A_{J}$ calculated within BAMPS while employing the improved Gunion-Bertsch matrix element together with a running coupling.

Fig. 2 shows the nuclear modification factor $R_{A A}$ for gluons, light quarks and pions/hadrons at RHIC and LHC obtained by BAMPS with PYTHIA [27] initial conditions together with a MonteCarlo Glauber sampling as described in detail in Ref. [10, 28]. It shows that gluons are stronger suppressed than light quarks over the whole $p_{t}$ range due to their larger QCD color factor. For comparison with data, we also show the $R_{A A}$ resulting from fragmentation via $\mathrm{AKK}$ fragmentation functions [29] for neutral pions and charged hadrons, respectively. A LPM parameter $X_{L P M}=0.3$ shows the best agreement with RHIC data and is therefore fixed to this value throughout the rest of this paper. With the fixed value we do not only describe the $R_{A A}$ at RHIC but also explain successfully the $R_{A A}$ for charged hadrons at LHC.

Hadrons at low $p_{t}$ are dominated by fragmenting gluons, while at higher $p_{t}$ the quark contribution increases. This effect together with the rising shape of the $R_{A A}$ leads to a hadronic $R_{A A}$ that is close to the quark $R_{A A}$. Since the shape of the partonic $R_{A A}$ at LHC is steeper than at RHIC, this effect is even more pronounced. 

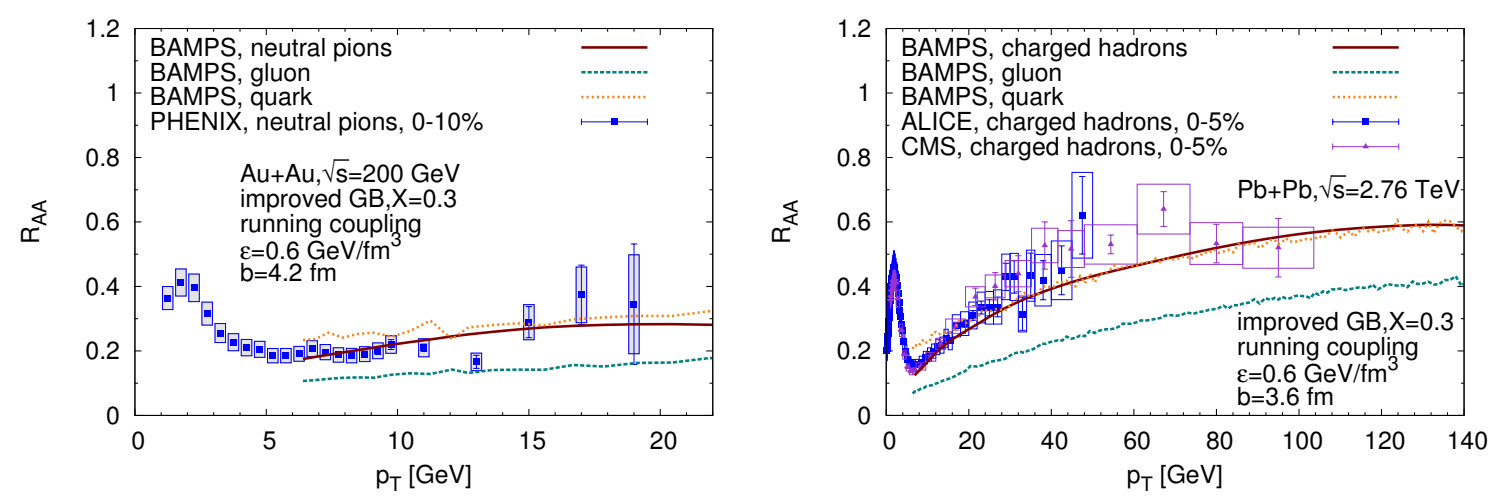

Figure 2: Nuclear modification factor $R_{A A}$ of gluons, light quarks, and neutral pions at RHIC (left) and charged hadrons at LHC (right), respectively, for a running coupling and LPM parameter $X=0.3$ together with data of neutral pions [25] and charged hadrons [26], respectively.



Figure 3: Momentum imbalance $A_{J}$ by BAMPS with PYTHIA initial conditions and CMS trigger conditions for a running coupling and LPM parameter $X=0.3$ together with data by CMS [5]. Additionally, the initial $A_{J}$ distribution obtained by PYTHIA is shown.

By using PYTHIA initial conditions and the improved GB matrix element together with a running coupling and the fixed $X_{L P M}=0.3$ parameter, Fig. 3 shows the momentum imbalance calculated within BAMPS together with data by CMS [5]. After employing the trigger conditions given by CMS and reconstructing the jets by the anti- $k_{t}$ algorithm as implemented in the package FASTJET [30], BAMPS shows a good agreement with data. As a remark, since the data is not corrected for the finite experimental jet energy resolution a Gaussian smearing for the reconstructed jet momenta is applied based on a comparison between PYTHIA simulations and the measured $\mathrm{p}+\mathrm{p}$ $A_{J}$ distribution as shown in Fig. 3. For more details and information about the simulation strategy of reconstructed jets within BAMPS and especially the complications arising from the employed test-particle ansatz, we refer to Ref. [31]. 

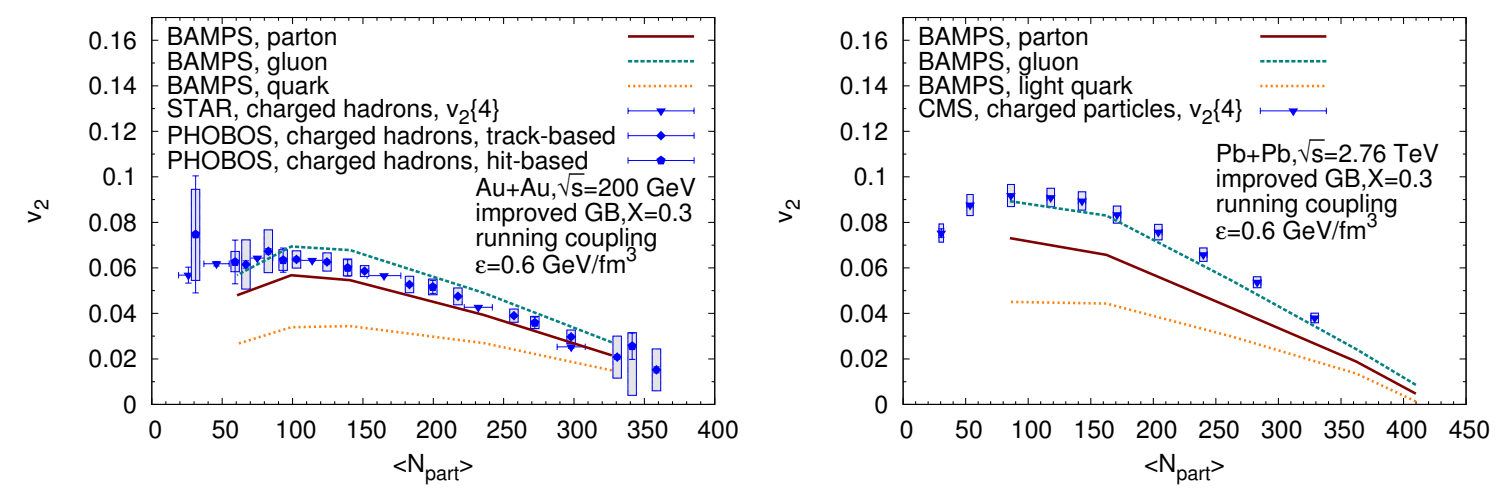

Figure 4: Elliptic flow $v_{2}$ of gluons, light quarks, and both together (light partons) within $|\eta|<1.0$ at RHIC (left) and $|\eta|<0.8$ at LHC (right) as a function of the number of participants $N_{\text {part }}$ for a running coupling and LPM parameter $X=0.3$. As a comparison we show experimental data by STAR and PHOBOS for charged hadrons within $|\eta|<0.5$ and $|\eta|<1.0$ (left) [32,33] and CMS for charged hadrons within $|\eta|<0.8$ (right) [34].

After presenting the results on the quenching of jets, we show the results for the bulk evolution by employing the same setup as already described above together with the fixed LPM parameter $X_{L P M}=0.3$ and a freeze-out energy density $\varepsilon_{c}=0.6 \mathrm{GeV} / \mathrm{fm}$ [20]. Since the microscopic hadronization processes within the soft regime are not fully understood yet, we show in fig. 4 our results for the integrated, partonic $v_{2}$ as a function of the number of participants $N_{\text {part }}$ in comparison with RHIC and LHC data. Assuming a coalescence picture, it can be shown that the partonic and hadronic integrated $v_{2}$ are supposed to be identical.

Remarkably, by using the same microscopic pQCD interactions for both the hard and the soft momentum regime, BAMPS media build up a sizable amount of flow within the partonic phase: The difference of the integrated, partonic $v_{2}$ of BAMPS and the measured, hadronic $v_{2}$ both at RHIC and LHC is about $10-20 \%$. The gluonic contribution to the flow is again higher than the quark contribution due to the larger color factors resulting from the underlying pQCD interactions. Reasons for the remaining difference between BAMPS and data stems mainly from the missing fluctuations in the initial state as well as the missing hadronic interactions after freeze-out.

The sizable flow within BAMPS originates mainly from the isotropization via inelastic $2 \leftrightarrow 3$ processes. However, due to the running coupling this isotropization is dominantly at low $p_{t}$ where the coupling is large. At high $p_{t}$, where jets are suppressed, the coupling is significantly smaller. This allows the description of $R_{A A}$ and, at the same time, the building of a sizable flow within the medium regime by using the same microscopic interactions.

\section{Conclusions}

While using full 3+1D microscopic simulations obtained by the partonic transport model BAMPS, we presented our recent progress in understanding the QGP in terms of the nuclear modification factor $R_{A A}$, the momentum imbalance $A_{J}$ of reconstructed di-jets and the elliptic flow $v_{2}$. By employing an improved Gunion-Bertsch matrix element, which gives a better agreement with the exact pQCD matrix element at forward and backward gluon rapidity, and a running coupling 
evaluated at the microscopic level, we are able to describe the jet quenching in terms of $R_{A A}$ and $A_{J}$ at RHIC and LHC, respectively. Furthermore, the same microscopic interactions lead to a sizable elliptic flow of the bulk medium. In contrast to previous BAMPS simulations, where the original GB approximation and a fixed coupling was used, the running coupling lead to an isotropization in the bulk regime while providing a realistic suppression at higher $p_{t}$.

For further understanding the energy loss of high $p_{t}$ particles and especially its path-length dependence we are currently investigating the implementation of a stochastic LPM effect similar to $[35,36]$. This would allow further constraints for the implemented LPM parameter $X_{L P M}$. Furthermore, to quantitatively describe the missing contribution to the elliptic flow $v_{2}$, we plan to implement initial state fluctuations in the primary nucleon-nucleon collisions. This would additionally allow the study of higher Fourier coefficients like e.g. $v_{3}$. Moreover, we are currently investigating the correlations of heavy flavor [37,38] within the same framework.

\section{A. Acknowledgments}

This work was supported by the Bundesministerium für Bildung und Forschung (BMBF), HGS-HIRe, H-QM, and the Helmholtz International Center for FAIR within the framework of the LOEWE program launched by the State of Hesse. Numerical computations have been performed at the Center for Scientific Computing (CSC).

\section{References}

[1] B. V. Jacak and B. Müller, Science 337, 310 (2012).

[2] B. Müller, J. Schukraft, and B. Wyslouch, Ann.Rev.Nucl.Part.Sci. 62, 361 (2012), 1202.3233.

[3] ATLAS Collaboration, G. Aad et al., Phys.Rev.Lett. 105, 252303 (2010), 1011.6182.

[4] CMS Collaboration, S. Chatrchyan et al., Phys.Rev. C84, 024906 (2011), 1102.1957.

[5] CMS Collaboration, S. Chatrchyan et al., Phys.Lett. B712, 176 (2012), 1202.5022.

[6] K. Geiger and B. Müller, Nucl.Phys. B369, 600 (1992).

[7] B. Zhang, Comput.Phys.Commun. 109, 193 (1998), nucl-th/9709009.

[8] D. Molnar and M. Gyulassy, Phys.Rev. C62, 054907 (2000), nucl-th/0005051.

[9] S. Bass, B. Müller, and D. Srivastava, Phys.Lett. B551, 277 (2003), nucl-th/0207042.

[10] Z. Xu and C. Greiner, Phys.Rev. C71, 064901 (2005), hep-ph/0406278.

[11] Z.-W. Lin, C. M. Ko, B.-A. Li, B. Zhang, and S. Pal, Phys.Rev. C72, 064901 (2005), nucl-th/0411110.

[12] M. Ruggieri, F. Scardina, S. Plumari, and V. Greco, Phys.Lett. B727, 177 (2013), 1303.3178.

[13] Z. Xu and C. Greiner, Phys.Rev. C76, 024911 (2007), hep-ph/0703233.

[14] F. A. Berends, R. Kleiss, P. De Causmaecker, R. Gastmans, and T. T. Wu, Phys.Lett. B103, 124 (1981).

[15] J. Gunion and G. Bertsch, Phys.Rev. D25, 746 (1982).

[16] O. Fochler, J. Uphoff, Z. Xu, and C. Greiner, Phys.Rev. D88, 014018 (2013), 1302.5250. 
[17] J. Uphoff, O. Fochler, Z. Xu, and C. Greiner, Phys.Rev. C84, 024908 (2011), 1104.2295.

[18] J. Uphoff, O. Fochler, Z. Xu, and C. Greiner, Phys.Lett. B717, 430 (2012), 1205.4945.

[19] Z. Xu, C. Greiner, and H. Stöcker, Phys.Rev.Lett. 101, 082302 (2008), 0711.0961.

[20] Z. Xu and C. Greiner, Phys.Rev. C79, 014904 (2009), 0811.2940.

[21] Z. Xu and C. Greiner, Phys.Rev. C81, 054901 (2010), 1001.2912.

[22] O. Fochler, Z. Xu, and C. Greiner, Phys.Rev.Lett. 102, 202301 (2009), 0806.1169.

[23] O. Fochler, Z. Xu, and C. Greiner, Phys.Rev. C82, 024907 (2010), 1003.4380.

[24] O. Fochler, J. Uphoff, Z. Xu, and C. Greiner, J.Phys. G38, 124152 (2011), 1107.0130.

[25] PHENIX Collaboration, A. Adare et al., Phys.Rev.Lett. 101, 232301 (2008), 0801.4020.

[26] ALICE Collaboration, B. Abelev et al., Phys.Lett. B720, 52 (2013), 1208.2711.

[27] T. Sjostrand, S. Mrenna, and P. Skands, JHEP 05, 026 (2006), hep-ph/0603175.

[28] J. Uphoff, O. Fochler, Z. Xu, and C. Greiner, Phys.Rev. C82, 044906 (2010), 1003.4200.

[29] S. Albino, B. Kniehl, and G. Kramer, Nucl.Phys. B803, 42 (2008), 0803.2768.

[30] M. Cacciari, G. P. Salam, and G. Soyez, Eur.Phys.J. C72, 1896 (2012), 1111.6097.

[31] F. Senzel, O. Fochler, J. Uphoff, Z. Xu, and C. Greiner, (2013), 1309.1657.

[32] STAR Collaboration, J. Adams et al., Phys.Rev. C72, 014904 (2005), nucl-ex/0409033.

[33] PHOBOS Collaboration, B. Back et al., Phys.Rev. C72, 051901 (2005), nucl-ex/0407012.

[34] CMS Collaboration, S. Chatrchyan et al., Phys.Rev. C87, 014902 (2013), 1204.1409.

[35] K. Zapp, J. Stachel, and U. A. Wiedemann, Phys.Rev.Lett. 103, 152302 (2009), 0812.3888.

[36] C. E. Coleman-Smith and B. Müller, Phys.Rev. C86, 054901 (2012), 1205.6781.

[37] J. Uphoff, F. Senzel, Z. Xu, and C. Greiner, (2013), 1310.1340.

[38] R. Abir, C. Greiner, M. Martinez, M. G. Mustafa, and J. Uphoff, Phys.Rev. D85, 054012 (2012), 1109.5539. 